\title{
Evolution of Progenitor Stars of Type Ibc Supernovae and Long Gamma-Ray Bursts
}

\author{
Sung-Chul Yoon ${ }^{1}$, Norbert Langer ${ }^{2}$, Matteo Cantiello $^{2}$, \\ Stan E. Woosley ${ }^{1}$ and Gary A. Glatzmaier ${ }^{3}$ \\ ${ }^{1}$ Department of Astronomy \& Astrophysics, University of California, Santa Cruz \\ High Street, Santa Cruz, CA 95064, USA \\ ${ }^{2}$ Astronomical Institute, Utrecht University, Utrecht, The Netherlands \\ 3Department of Earth \& Planetary Sciences, University of California, Santa Cruz \\ High Street, Santa Cruz, CA 95064, USA
}

\begin{abstract}
We discuss how rotation and binary interactions may be related to the diversity of type Ibc supernovae and long gamma-ray bursts. After presenting recent evolutionary models of massive single and binary stars including rotation, the Tayler-Spruit dynamo and binary interactions, we argue that the nature of SNe Ibc progenitors from binary systems may not significantly differ from that of single star progenitors in terms of rotation, and that most long GRB progenitors may be produced via the quasi-chemically homogeneous evolution at sub-solar metallicity. We also briefly discuss the possible role of magnetic fields generated in the convective core of a massive star for the transport of angular momentum, which is potentially important for future stellar evolution models of supernova and GRB progenitors.
\end{abstract}

Keywords. stars: evolution - binaries: close - stars: magnetic fields - stars: rotation - supernovae: general

\section{Introduction}

Rotation influences not only the evolution of massive stars, but also their supernova (SN) explosions (Maeder \& Meynet 2000; Heger, Langer \& Woosley 2000). In particular, recently many asymmetric supernovae with unusually large energy (broad-lined SNe or hypernovae) have been discovered (e.g. Mazzali et al. 2007), which shows evidence for rapidly rotating progenitors. The most spectacular example may be long gamma-ray bursts (GRBs), which are generally believed to be produced by deaths of some massive stars retaining extremely large angular momenta in their cores $\left(j \geqslant \sim 10^{16} \mathrm{~cm}^{2} \mathrm{~s}^{-1}\right.$; Woosley 1993; MacFadyen \& Woosley 1999; see, however, Dessart et al. 2008). Interestingly, such energetic core-collapse events seem to only occur in Wolf-Rayet (WR) stars: all of the broad-lined SNe/Hypernovae and the supernovae associated with long GRBs have been observationally identified as Type Ic (see Woosley \& Bloom 2006 for a review). This raises the question which WR stars can produce broad-lined SNe Ic or long GRBs while most WR stars die as normal SNe Ibc. Here we present recent evolutionary models of massive stars that include binary interactions and the transport of chemical species and angular momentum via rotationally induced hydrodynamic instabilities and magnetic torques, and discuss how rotation and binary interactions may be related to the diversity of SNe Ibc and long GRBs.

\section{Single star models}

Redistribution of angular momentum and chemical species in a rotating star occurs by rotationally induced hydrodynamic instabilities and magnetic torques (See Talon 2007 

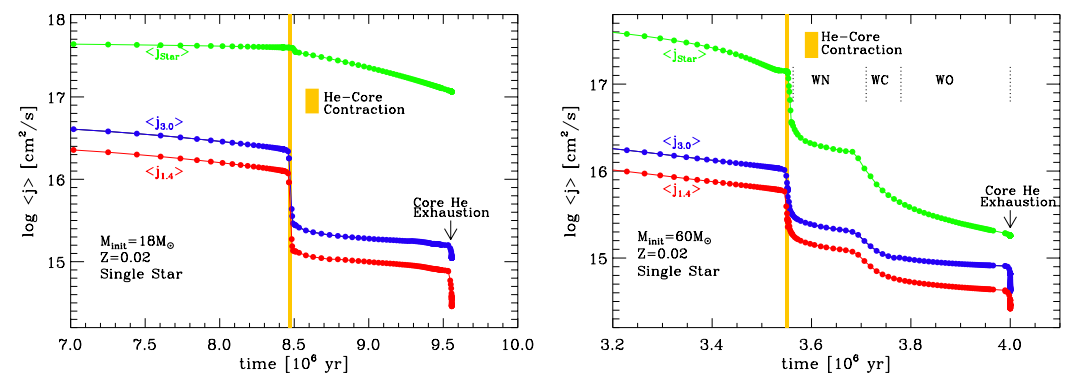

Figure 1. Mean specific angular momentum of the star and the innermost $1.4 \mathrm{M}_{\odot}$ and $3.0 \mathrm{M}_{\odot}$ as a function of the evolutionary time for $18 \mathrm{M}_{\odot}$ (left panel) and $60 \mathrm{M}_{\odot}$ (right panel) models. The time span for the helium core contraction is marked by the color shade as indicated by the label.

for a review). Eddington-Sweet circulations, shear instability and Goldreich-SchubertFricke instability among others have been considered in previous non-magnetic models (Maeder \& Meynet 2000; Heger, Langer \& Woosley 2000; Hirschi, Meynet \& Maeder 2004), and the so-called Tayler-Spruit dynamo (Spruit 2002) has been implemented in recent magnetic models (e.g. Heger, Woosley \& Spruit 2005; Maeder \& Meynet 2005; Yoon \& Langer 2005). In non-magnetic models, it is shown that the buoyancy due to the chemical gradient at the interface between the core and the envelope largely prohibits the considered rotationally induced hydrodynamic instabilities from transporting angular momentum. The amount of angular momentum retained in the core at the pre-supernova stage is thus close to its initial value even at solar metallicity (Heger, Langer \& Woosley 2000; Hirschi, Meynet \& Maeder 2004). Most massive stars are predicted to die with an enough amount of angular momentum in the cores to produce long GRBs via formation of millisecond magnetars or collapsar (i.e., $j \geqslant \sim 10^{16} \mathrm{~cm}^{2} \mathrm{~s}^{-1}$ ), given that a large fraction of young massive stars in our Galaxy and Small/Large Magellanic Clouds are rapid rotators (e.g. Maeder \& Meynet 2000; Mokiem et al. 2006; Hunter et al. 2008). On the other hand, in magnetic models adopting the Tayler-Spruit dynamo the core is effectively spun down by magnetic torques, and the predicted spin rates of white dwarfs and young neutron stars are smaller by two orders of magnitude than those from non-magnetic models, which can better explain observations (Heger, Woosley \& Spruit 2005; Suijs et al. 2008).

Fig. 1 shows the evolution of the core angular momentum in the magnetic model sequences with $M_{\text {init }}=18 \mathrm{M}_{\odot} \& v_{\text {rot,init }}=144 \mathrm{~km} \mathrm{~s}^{-1}$, and $60 \mathrm{M}_{\odot} \& v_{\text {rot,init }}=$ $186 \mathrm{~km} \mathrm{~s}^{-1}$. In the sequence with $M_{\text {init }}=18 \mathrm{M}_{\odot}$, the core loses a significant amount of angular momentum during the helium core contraction phase where a strong degree of differential rotation between the core and the envelope appears. A similar effect is also observed during the $\mathrm{CO}$ core contraction phase. In the sequence with $M_{\text {init }}=60 \mathrm{M}_{\odot}$, spinning-down of the core during He-core contraction becomes less significant as the star loses the hydrogen envelope, which leads to a smaller moment of inertia of the envelope. However, the core is further spun down by loss of mass due to LBV/WR winds during core He burning. At the neon burning stage, both stars retain a similar amount of angular momentum in their cores as shown in Fig. 1. In fact, the calculations by Yoon, Langer \& Norman (2006) show that magnetic models give $<j_{1.4}>\simeq 2 \ldots 3 \times 10^{14} \mathrm{~cm}^{2} \mathrm{~s}^{-1}$ for different initial metallicities, masses and rotational velocities, implying that most massive stars including Type Ibc progenitors should die with a similar amount of core angular momentum. This conclusion remains the same even for binary stars, as discussed below. 

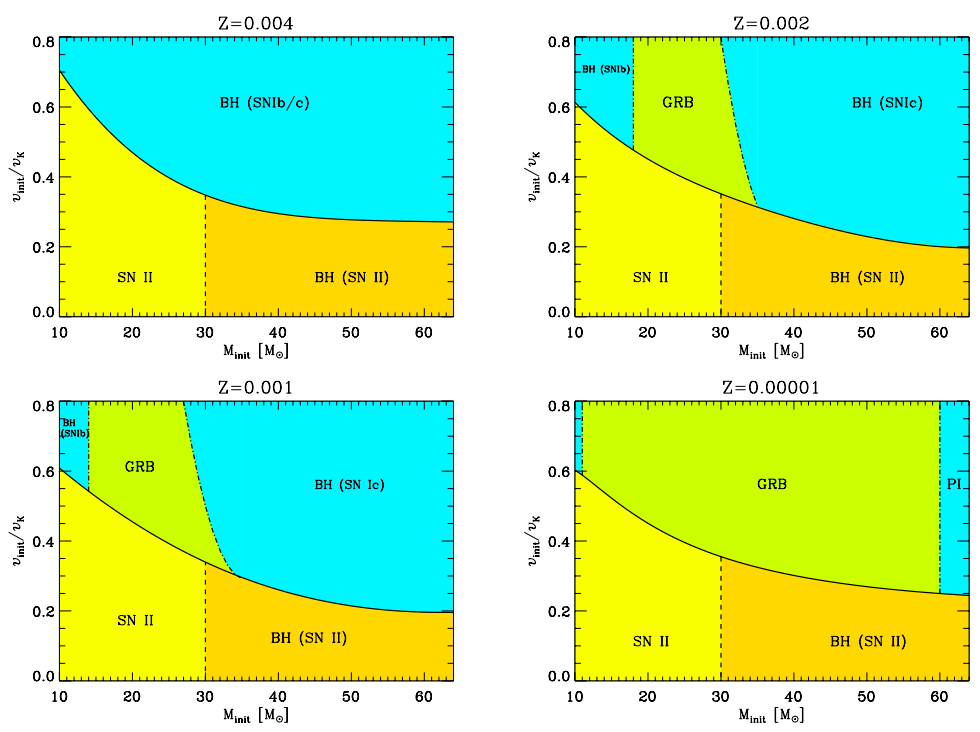

Figure 2. Final fate of our rotating massive star models at four different metallicities $(Z=$ $0.004,0.002,0.001, \& 0.00001)$, in the plane of initial mass and initial fraction of the Keplerian value of the equatorial rotational velocity. The solid line divides the plane into two parts, where stars evolve quasi-chemically homogeneous above the line, while they evolve into the classical core-envelope structure below the line. The dotted-dashed lines bracket the region of quasi-homogeneous evolution where the core mass, core spin and stellar radius are compatible with the collapsar model for GRB production (absent at $\mathrm{Z}=0.004$ ). To both sides of the GRB production region for $Z=0.002$ and 0.001 , black holes are expected to form inside WR stars, but the core spin is insufficient to allow GRB production. For $Z=0.00001$, the pair-instability might occur to the right side of the GRB production region, although the rapid rotation may shift the pair instability region to larger masses. The dashed line in the region of non-homogeneous evolution separates Type II supernovae (SN II; left) and black hole (BH; right) formation, where the minimum mass for $\mathrm{BH}$ formation is simply assumed to be $30 \mathrm{M}_{\odot}$. From Yoon, Langer \& Norman (2006).

An exception is the case for the so-called chemically homogeneous evolution. If the initial rotational velocity is exceptionally high and if metallicity is sufficiently low, chemical mixing by Eddington-Sweet circulations may occur on a time scale even smaller than the nuclear time scale. Quasi-homogeneity of the chemical composition of a star is thus ensured on the main sequence and the star is gradually transformed into a massive WR star, avoiding the giant phase that would result in strong braking down of the core. All of the necessary conditions for producing long GRBs - massive core to make a black hole, removal of hydrogen envelope and retention of a large amount of angular momentum in the core - thus can be fulfilled by this type of evolution (Yoon \& Langer 2005; Woosley \& Heger 2006). This chemically homogeneous evolution scenario (CHES) favors low metallicity environment for producing long GRBs (Fig. 2) and predicts a higher ratio of GRB to SN rate at higher redshift, which should be tested by future observations (Yoon, Langer \& Norman 2006; cf. Kistler et al. 2008).

\section{Binary star models}

A significant fraction of SNe Ibc may be produced in close binary systems (e.g. Podsiadlowski, Joss \& Hsu 1992). An example is given in Fig. 3 that shows the evolution of of the primary star in a close binary system with $P_{\text {init }}=4$ days, $M_{\text {primary, init }}=18 \mathrm{M}_{\odot}$, and 

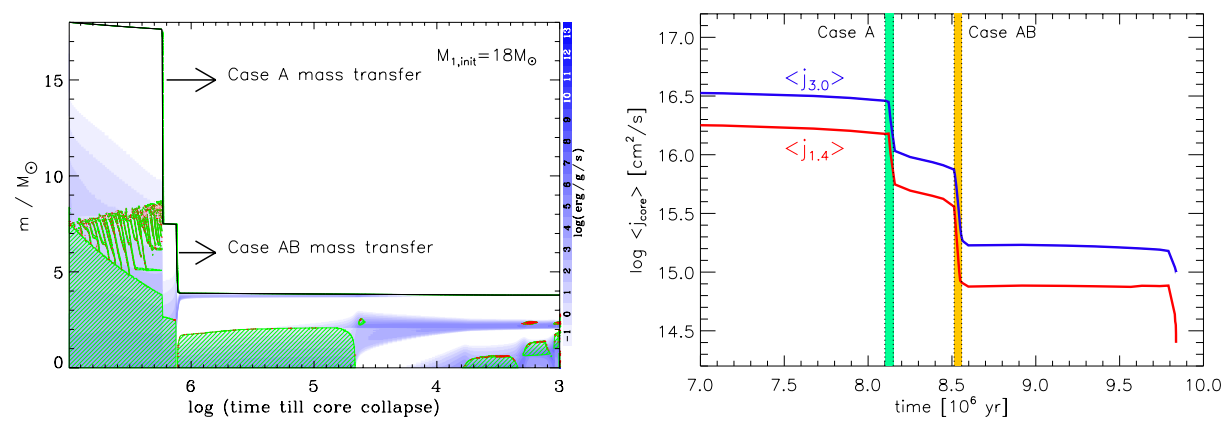

Figure 3. Left panel Evolution of the internal structure of the primary star in a binary system of $M_{\text {primary,init }}=18 \mathrm{M}_{\odot}, M_{\text {secondary, init }}=17 \mathrm{M}_{\odot}$ and $P_{\text {init }}=4$ days, from zero age main sequence to neon burning. Right panel Mean specific angular momentum of the innermost $1.4 \mathrm{M}_{\odot}$ and $3.0 \mathrm{M}_{\odot}$ of the primary star considered in the left panel as a function of time. The time span for Case A or Case AB mass transfer phase is marked by the color shades as indicated by the labels.

$M_{\text {secondary,init }}=17 \mathrm{M}_{\odot}$. Both stars are tidally synchronized early on the main sequence. Once the primary star fills the Roche-lobe radius, it loses about $7 \mathrm{M}_{\odot}$ during the Case A mass transfer phase, and additional $3.5 \mathrm{M}_{\odot}$ later during the Case $\mathrm{AB}$ mass transfer phase, becoming a $4 \mathrm{M}_{\odot}$ WR star. The core loses angular momentum mostly during these Case $\mathrm{A}$ and $\mathrm{AB}$ mass transfer phases as shown in Fig. 3. The amount of angular momentum in the core at the neon burning stage turns out to be very similar to those in single star models (see Fig. 1). In fact, we find that mean specific angular momentum of the innermost $1.4 \mathrm{M}_{\odot}$ at the final evolutionary stage of a primary star in a binary system does not change much according to different initial parameters (primary mass, mass ratio and orbital separation): it remains within a narrow range of $2 \ldots 3.5 \times 10^{14} \mathrm{~cm}^{2} \mathrm{~s}^{-1}$ regardless of the detailed history of binary interactions, as long as the tidal synchronization is not unusually strong (Yoon, Woolsey \& Langer 2008, in prep.). This implies that the nature of most binary star progenitors of SNe Ibc may not much differ from that of single star progenitors, in terms of rotation.

The evolution of secondary stars has not yet been well understood. In particular, it sensitively depends on the uncertain efficiency of semi-convection whether the mass accreting star may be rejuvenated or not (Braun \& Langer 1995). If a rather large semiconvection parameter is adopted, rejuvenation can significantly weaken the chemical gradient between the hydrogen burning core and the envelope, in favor of rotationally induced chemical mixing. As the secondary is spun up to the critical rotation by mass accretion, even the chemically homogeneous evolution can be occasionally induced if metallicity is sufficiently low and if the secondary is not strongly spun down by the tidal synchronization after the mass accretion phase (Cantiello et al. 2007). The secondary will eventually die as a GRB after traveling from a few to several hundreds PCs away, if the binary system is unbound due to the supernova kick as a result of the explosion of the primary. This scenario may explain the recent observational evidence that some GRBs are produced in runaway stars (Hammer et al. 2006).

Other types of binary interactions may also lead to formation of rapidly rotating WR stars to produce long GRBs. Tidal spinning-up of a WR star in a compact binary system with a neutron star or a black hole companion (Brown et. al. 2000; Izzard, RamirezRuiz \& Tout 2004; van Putten, M. H.P. M. 2004; van den Heuvel \& Yoon 2007) and merger of two helium cores in a common evenlope (Fryer \& Heger 2005) have been recently suggested among others. It remains uncertain, however, that such binary systems 


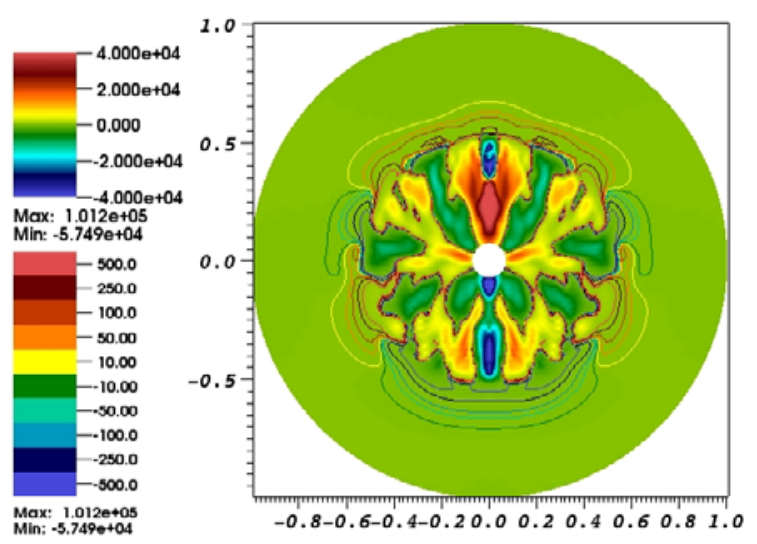

Figure 4. Mean radial fields $B_{\mathrm{r}}(r, \theta)$ on the meridional plane in a $12 \mathrm{M}_{\odot}$ rotating star on the main sequence in a MHD simulation with a 3-D anelastic code (Glatzmaier 1984). The adopted angular velocity is $10^{-5} \mathrm{Rad} \mathrm{s}^{-1}$. The inner region of $r \leqslant 7 \times 10^{10} \mathrm{~cm}(r \leqslant 0.5$ in the code units) is the convective core.

could explain the observed GRB rate. For example, recent stellar evolution models by Detmers, Langer \& Podsiadlowski (2008) show that a merger of the WR star with the compact object, which is not supposed to produce a classical long GRB, is the most likely outcome in the former case. Further detailed evolutionary models are certainly needed for observationally testing different evolutionary scenarios (e.g. van Marle et al. 2008). On the other hand, it is puzzling why no GRB-associated SNe Ib have been observed yet while most GRB progenitor scenarios predict their existence (e.g. Yoon, Langer \& Norman 2006). Within the CHES, this puzzle might be solved if one considered anisotropic mass loss, as discussed in Meynet \& Maeder (2007).

\section{Concluding remarks}

Our stellar evolution models including the Tayler-Spruit dynamo indicate that most SNe Ibc progenitors should explode with a similar amount of angular momentum in their cores, regardless of their single or binary star origin. This is due to the self-regulationary nature of the Tayler-Spruit dynamo. Loss of the hydrogen envelope due to stellar winds or mass transfer results in both removal of angular momentum from the core and weakening of the core braking by the extended hydrogen enveloped due to magnetic torques, and vice versa. Therefore, most different pre-supernova evolutionary paths may not contribute much to the diversity of Type Ibc supernovae in terms of core angular momentum, although different iron core masses, and thus different spin rates of young neutron stars may result (Heger, Woosley \& Spruit 2005). GRB progenitors, which require unusually large angular momenta, may undergo the chemically homogeneous evolution, which may not be unusual at low metallicity. On the other hand, recent observations indicate that not all broad-lined SNe Ic are associated with long GRBs (e.g. Modjaz et al. 2008), which needs a theoretical explanation in future work.

Although the predicted spin rates of the stellar remnants from the magnetic models are consistent with observations, the validity of the Tayler-Spruit dynamo has been recently questioned by several authors (Denissenkov \& Pinsonneault 2007; Zahn, Brun \& Mathis 2008). Furthermore, we might have ignored potentially important physical ingredients in simulating the evolution of rotating massive stars. These include gravity waves (Townsend in this volume), and magnetic fields generated by the convective core. For instance, 
our recent 3-D simulations with an anelastic magnetohydrodynamics code (Glatzmaier 1984) show that the strength of poloidal fields generated in the convective core in a young massive star may amount to several thousand Gauss on average (Fig. 4), and its influence on the transport processes might be comparable to what the Tayler-Spruit dynamo predicts. This issue will be addressed in Yoon, Woosely \& Glatzmaier (2008, in prep.).

\section{Acknowledgements}

This work was, in part, supported by the DOE Program for Scientific Discovery through Advanced Computing and NASA.

\section{References}

Braun, H. \& Langer, N. 1995, A\& A, 297, 483

Brown, G. E., Lee, C.-H., Wijers, R. A. M. J., et al. 2000, NewA, 5, 191

Cantiello, M., Yoon, S.-C., Langer, N., \& Livio, M. 2007, A $\& A$, 465, L29

Denissenkov, P. A. \& Pinsonneault, M. 2007, ApJ, 655, 1157

Dessart, L., Burrows, A., Livne, E., \& Ott, C. 2008, ApJL, 673, 43

Detmers, R., Langer, N., Podsiadlowski, Ph., \& Izzard, R. G. 2008, $A \& A$, submitted astro$\mathrm{ph} / 0804.0014$

Fryer, C. L. \& Heger, A. 2005, ApJ, 623, 302

Glatzmaier, G. A. 1984, J. Comp. Phys., 55, 461

Hammer, F., Flores, H., Schaerer, D., et al. 2006, A\& A, 454, 103

Heger, A., Langer, N., \& Woosley, S. E. 2000, ApJ,

Heger, A., Woosley, S. E., \& Spruit, H. C. 2005, ApJ, 626, 350

van den Heuvel, E. P. J \& Yoon, S.-C. 2007, Ap\&SSS, 311, 177

Hirschi, R., Meynet, G., \& Maeder, A. 2005, A\&A, 425, 649

Hunter, I, Lennon, D. J., Dufton, P. I., et al. 2008, A\& $A, 479,541$

Izzard, R. G., Ramirez-Ruiz, E., \& Tout, C. A. 2004, MNRAS, 348, 1215

Kistler, M. D., Yüksel, H., Beacom, J. F., \& Stanek, K. Z. 2008, ApJ, 673, L119

MacFadyen, A. I. \& Woosley, S. E. 1999, ApJ, 524, 262

Maeder, A. \& Meynet, G. 2000, ARA\&A, 38, 143

Maeder, A. \& Meynet, G. 2005, A\&SA, 440, 104

Mazzali, P. A., Kawabata, K. S., Maeda, K., et al. 2007, ApJ, 670, 592

Meynet, G. \& Maeder, A. 2007, A\&A, 464, L11

van Marle, A. J., Langer, N., Yoon, S.-C., \& Garcia-Segura, G. 2008, A\& A, 478, 769

Modjaz, M, Kewley, L., Kirshner, R. P., et al. 2008, AJ, 135, 1136

Mokiem, M. R., de Koter, A., Evans, C. J., et al. 2006, A\& A, 456, 1131

Podsiadlowski, Ph., Joss, P. C., \& Hsu, J. J.L. 1992, ApJ, 391, 246

van Putten, M. H. P. M. 2004, ApJL, 611, 81

Suijs, M., Langer, N., Poelarends, A. J., et al. 2008, A\&A, in prep.

Spruit, H. C. 2002, AESA, 381, 923

Talon, S. 2007, astro-ph/0708.1499

Woosley, S. E. 1993, ApJ, 405, 273

Woosley, S. E. \& Bloom, J. S. 2006, ARAधA, 44, 507

Woosley, S. E. \& Heger, A. 2006, ApJ, 637, 914

Yoon, S.-C. \& Langer, N. 2005, A\&BA,

Yoon, S.-C., Langer, N., \& Norman, C. 2006, A\&A, 460, 199

Zahn, J.-P., Brun, A. S., \& Mathis, S. 2007, A\&\&A, 474, 145 